\title{
The source of tropospheric tides
}

\author{
Xing Li 1,2,3,4* WeiXing Wan 1,4,5,6, JinBin Cao ${ }^{2,3}$, and ZhiPeng Ren ${ }^{1,4,5,6}$ \\ ${ }^{1}$ Key Laboratory of Earth and Planetary Physics, Institute of Geology and Geophysics, Chinese Academy of Sciences, Beijing 100029, China; \\ ${ }^{2}$ School of Space and Environment, Beihang University, Beijing 100083, China; \\ ${ }^{3}$ Key Laboratory of Space Environment Monitoring and Information Processing, Ministry of Industry and Information Technology, Beijing 100083, China; \\ ${ }^{4}$ Beijing National Observatory of Space Environment, Institute of Geology and Geophysics, Chinese Academy of Sciences, Beijing 100029, China; \\ 5 Innovation Academy for Earth Science, Chinese Academy of Sciences, Beijing 100029, China; \\ 6University of the Chinese Academy of Sciences, Beijing 100049, China
}

\section{Key Points:}

- The tidal sources of DW1 (diurnal westward propagating wavenumber 1), SW2 (semidiurnal westward propagating wavenumber 2), and DE3 (diurnal eastward propagating wavenumber 3) were obtained from the National Centers for Environmental Prediction (NCEP) Climate Forecast System Reanalysis (CFSR) data

- These tidal sources show obvious latitudinal and yearly variations

- Some propagating Hough modes dominate the tropospheric tides

Citation: Li, X., Wan, W. X., Cao, J. B., and Ren, Z. P. (2020). The source of tropospheric tides. Earth Planet. Phys., 4(5), 449-460. http://doi.org/10.26464/epp2020049

\begin{abstract}
With the method of Hough mode decomposition (HMD), the tidal sources of the three main tidal components, namely, the migrating components DW1 (diurnal westward propagating wavenumber 1) and SW2 (semidiurnal westward propagating wavenumber 2) and the non-migrating component DE3 (diurnal eastward propagating wavenumber 3 ), at the tropospheric altitudes (1-12 km) and in the latitude range of $\pm 60^{\circ}$, were obtained from National Centers for Environmental Prediction (NCEP) Climate Forecast System Reanalysis (CFSR) data during the interval from 1988 to 2011 . We analyzed these sources in detail at $6 \mathrm{~km}$ and obtained the main properties of their yearly variations. The DW1 source was found to present a weak seasonal variation in the lower latitudes (about $\left.\pm 10^{\circ}-15^{\circ}\right)$. That is, the amplitudes of the DW1 sources were larger in the summer months than in the winter months, and DW1 presented semi-annual variation near the equator $\left( \pm 10^{\circ}\right)$ such that the DW1 source was larger at the equinoxes than at the solstices. In addition, the SW2 source was symmetric and was stronger in the southern hemisphere than in the northern hemisphere. The SW2 source presented remarkable annual and semi-annual variation such that the amplitudes were largest during the March equinox months and larger during the June solstice months. In contrast, DE3 appeared mainly around the equatorial latitudes within about $\pm 30^{\circ}$. The DE3 source presented remarkable semiannual variation that was larger around the solstices than the equinoxes in the southern hemisphere, and it was opposite in the northern hemisphere. By HMD, we found that the tropospheric tides were primarily dominated by some leading propagating Hough modes, specifically, the $(1,1),(2,3)$, and $(3,3)$ modes; the influences of the other Hough modes were negligible. The consequences of an El Niño-Southern Oscillation modulation of tidal amplitudes for the energy and momentum budgets of the troposphere may now be expected to attract attention. In summary, the above yearly variations of the main tidal sources and the Hough coefficients demonstrate that an HMD analysis can be used to investigate the tropospheric tides.
\end{abstract}

Keywords: tropospheric tides; Hough mode decomposition; yearly variations

\section{Introduction}

The atmospheric solar tides are important global-scale waves whose periods are not longer than a solar day. The tidal functions may vary with time, latitude, and longitude, and their frequencies ( $k$ in numbers/day) and zonal wavenumbers ( $f$ in numbers/cycle) must be integers. We often divide tides into "migrating tides" and "non-migrating tides." The migrating tide functions $(f=-k)$

Correspondence to: X. Li, lixing@buaa.edu.cn

Received 02 APR 2020; Accepted 02 JUN 2020.

Accepted article online 20 JUL 2020.

(C) 2020 by Earth and Planetary Physics. mainly vary with local time and do not depend on longitude; in contrast, the non-migrating tides $(f \neq-k)$ depend on both local time and longitude (Chapman and Lindzen, 1970). Usually, diurnal westward propagating wavenumber 1 , or DW1 $(f=1, k=-1)$, and semidiurnal westward propagating wavenumber 2 , or SW2 $(f=2, k=-2)$, are the main migrating components, and diurnal eastward propagating wavenumber 3 , or DE3 $(f=1, k=3)$, is the most important non-migrating component (e.g., Talaat and Lieberman, 1999; Manson et al., 2002; Forbes et al., 2003; Forbes and Wu D, 2006; Pancheva et al., 2010).

Chapman and Lindzen (1970) described various sources of tidal excitation. It was demonstrated that the insolation absorption by 
ozone and water vapor is the most important source of thermal excitation below 70 km (e.g., Lindzen and Blake, 1970; Forbes and Garrett, 1979; Hagan, 1996; Hagan and Forbes, 2002). Harris and Mayr (1975), Forbes and Garrett (1978), and Mayr and Harris (1977) observed that the atmospheric thermal tides are excited by ultraviolet and extreme ultraviolet absorption in the lower thermosphere (90-200 km). In addition, Lindzen and Will (1973) and Hamilton (1981) first identified the latent heat release by largescale deep convection as a possible source of tidal excitation.

Further research has shown a great many sources for the tidal components. Migrating tides are prompted primarily by the energy absorption of tropospheric water, water vapor, and stratospheric ozone (Oberheide et al., 2002). Non-migrating tides are excited by numerous sources, such as the land-sea differences, the zonal asymmetries into topography, the longitude dependencies in the absorbing species (Forbes et al., 2003), and the nonlinear interactions between different migrating components as well as between the migrating tides and other atmospheric waves (i.e., planetary waves; Hagan and Roble, 2001) or gravity waves (McLandress and Ward, 1994). It is known that the source of the tidal component is excited during our altitudinal range (0-12 km), though the amplitudes are relatively small there. The source plays an important role in the tidal component in the upper altitudes. Non-migrating tides may induce longitudinal variability in all the parameters in the middle and upper atmosphere (Hagan and Forbes, 2002; Li X et al., 2015a) and even ionosphere (Ren ZP et al., 2008; Wan W et al., 2008, 2010).

Assuming that the atmosphere is isothermal and motionless, the atmosphere leads to a separation between Laplace's tidal equation, which determines the latitudinal structure of tides, and a vertical structure equation, which connects the structure of tides by equivalent depths (eigenvalues; Forbes, 1995). The tidal structures are described in terms of the modal latitudinal structure (Hough modes) and vertical wavelengths (Chapman and Lindzen, 1970). Governing the global-scale tidal oscillations, the researchers involves a set of linearized and simplified primitive equations that illustrate the sources of the atmospheric tides.

The main purpose of the present work is to investigate the variability of the sources of the main tidal components (i.e., DW1, SW2, and DE3) in the troposphere, as reflected in the National Centers for Environmental Prediction (NCEP) Climate Forecast System Reanalysis (CFSR) data, and to unveil the global latitudinal structure of the main tidal components. In Section 2, we describe the reanalysis data and the analytical methods. In Section 3, we present the yearly variations of the main tidal sources. In Section 4 , we illustrate the yearly variation of the main tidal components. Additionally, we present evidence that the El Niño-Southern Oscillation (ENSO) is a source of tidal variability. Finally, we provide a summary and conclusions in Section 5.

\section{Data Set and Analytical Method}

Generated by a constant model and a frozen data assimilation system, the NCEP CFSR data sets produce multiyear, global state-ofthe-art gridded representations of atmospheric states (Saha et al., 2010). The data sets use a time resolution of 1 hour, a horizontal resolution of $2.5^{\circ}$ longitude by $2.5^{\circ}$ latitude, and a vertical grid spacing of five pressure levels $(1,000,850,700,500$, and $200 \mathrm{hPa})$, corresponding to an altitude of about $0-12 \mathrm{~km}$, over the period from 1979 to 2011. Fortunately, the longitude and time resolutions of the CFSR reanalysis data are sufficient to analyze the structures of most of the tidal components of the tropospheric parameters, including temperature, vector wind, and geopotential height. Li et al. (2015b) and Sakazaki et al. (2012) have also suggested that the CFSR data, as a supplementary observation, can be used in the study and modelling of atmospheric tides. The CFSR data were provided through the National Center for Atmospheric Research/University Corporation for Atmospheric Research Data Archive at http://rda.ucar.edu/datasets/ds093.1/.

In this work, the CFSR data from 1988 to 2011 were used to study the three main tidal components, DW1, SW2, and DE3. We chose the temperature $(T)$, geopotential height $(h)$, and zonal wind $(U)$ as the atmospheric parameters in the tidal analysis. Because, in the upper atmosphere, researchers often analyze the tides by atmospheric density, we transformed the geopotential height $(h)$ into density $(\rho)$ to compare them. In the data sets, each of these three parameters could be represented as a four-dimensional function of longitude $(\lambda)$, latitude $(\varphi)$, altitude $(z)$ or pressure level, and universal time $(t)$, or $T(\lambda, \varphi, z, t), \rho(\lambda, \varphi, z, t)$, and $U(\lambda, \varphi, z, t)$. By a Fourier fitting technique, the data in each latitude bin were decomposed into tidal models. Thus, in general, atmospheric tides could be expressed in the form

$$
\left\{\begin{array}{l}
T(\lambda, \varphi, z, t)=\sum_{f, k} A_{T}(f, k, \varphi, z) \cos \left(2 \pi\left(f\left(t-\Phi_{T}(f, k)\right)-k \lambda\right)\right), \\
\rho(\lambda, \varphi, z, t)=\sum_{f, k} A_{\rho}(f, k, \varphi, z) \cos \left(2 \pi\left(f\left(t-\Phi_{\rho}(f, k)\right)-k \lambda\right)\right), \\
U(\lambda, \varphi, z, t)=\sum_{f, k} A_{U}(f, k, \varphi, z) \cos \left(2 \pi\left(f\left(t-\Phi_{U}(f, k)\right)-k \lambda\right)\right) .
\end{array}\right.
$$

After working with the data sets, we obtained three-dimensional (3-D) discrete data on temperature, density, and zonal winds, which were present at certain altitudes during certain universal times $(t)$. To quantify the oscillations responsible for the tidal structure, we had to identify their individual contributions. We performed a two-dimensional (2-D) Fourier transform for every combination of latitude and altitude, thus revealing all the tidal and stationary planetary wave contributions.

After decomposing the atmospheric tidal components, we analyzed their propagation characteristics by using a Hough mode decomposition (HMD), which can be thought of as constraining a tidal model with observations. This produces self-consistent tidal fields in temperature, density, and zonal winds $(T, \rho, U)$. Classical atmospheric tidal wave models describe the eigenwave in a homogeneous, non-dissipative, and isothermal atmosphere. Hough modes represent the eigen-solution of Laplace's tidal equation to global-scale waves in the ideal background atmosphere. Under the assumption of non-dissipation, wave equations can be separated from variables. Recently, the HMD has also been used to decompose the atmospheric tidal components (Chen ZY and Lu DR, 2009a; Yu Y et al., 2013).

The Hough mode is labelled by a number of pairs, $\Theta(m, n)$, where $m$ denotes the zonal wavenumber of the atmospheric tides and $n$ 
is the Hough mode series number. The propagating modes represent the propagating waves, which are capable of propagating upward from the lower atmosphere, whereas the trapped modes denote waves that are locally excited and have no vertical propagation. We know that the bottom response is complex and is caused by more than one mechanism. Therefore, to understand the propagating mechanism from below, we utilized only the nine leading propagating modes in our decomposition. To the diurnal migrating tide DW1, the series number $n$ represented $1,2,3,4,5$, $6,7,8$, and 9, respectively; to the semidiurnal migrating tide SW2, the series number $n$ represented $2,3,4,5,6,7,8,9$, and 10 , respectively; and to the non-migrating tide DE3, the series number $n$ represented $3,4,5,6,7,8,9,10$, and 11 , respectively. In addition, Hough modes alternate between symmetric and antisymmetric relative to the equator; that is, they are symmetric (antisymmetric) when $m+n$ is even (odd). According to previous works (e.g., Svoboda et al., 2005; Forbes and Wu D, 2006; Zhang XL et al., 2006; Chen ZY and Lu DR, 2009a, b; Oberheide et al, 2009; Pancheva et al., 2010; Oberheide et al., 2011; Yu Y et al., 2015), Hough modes converge quickly, and only the weighted superposition of the few leading modes is required to represent the atmospheric tides. Figure 1 shows the normalized Hough modes as functions of latitude, and Table 1 shows the Lamb numbers (eigenvalues) of these modes.

In the atmosphere, HMD has been successful in providing good analytical results to a great extent (Chen ZY and Lu DR, 2009b; Yu $Y$ et al., 2013). The Hough function is an effective analytical tool used to research the global structure of atmospheric tides. In this work, the results from calculating the Hough functions based on Laplace's tidal equation were applied to atmospheric tides, and the overall features of the yearly variations and the complete global structure of the tides were studied. The approach used to conduct the HMD analysis can be expressed as follows:

$$
\left\{\begin{array}{l}
A_{T}(f, k, \varphi, z)=\sum_{n} C_{n}(z) \cdot A_{T \perp}(z, f, n) \cdot \Theta_{T}(f, k, \varphi, z), \\
A_{\rho}(f, k, \varphi, z)=\sum_{n} C_{n}(z) \cdot A_{\rho \perp}(z, f, n) \cdot \Theta_{\rho}(f, k, \varphi, z), \\
A_{U}(f, k, \varphi, z)=\sum_{n} C_{n}(z) \cdot A_{U \perp}(z, f, n) \cdot \Theta_{U}(f, k, \varphi, z),
\end{array}\right.
$$

where $n$ is the Hough mode series number; $\Theta$ is the Hough mode, which depends on the latitude $\varphi$ and $n ; C_{n}$ is the Hough coefficient, which depends on $z$; and $A_{\perp}$ is the vertical structure, which depends on $z, f$, and $n$. The equations were set to the temperature,
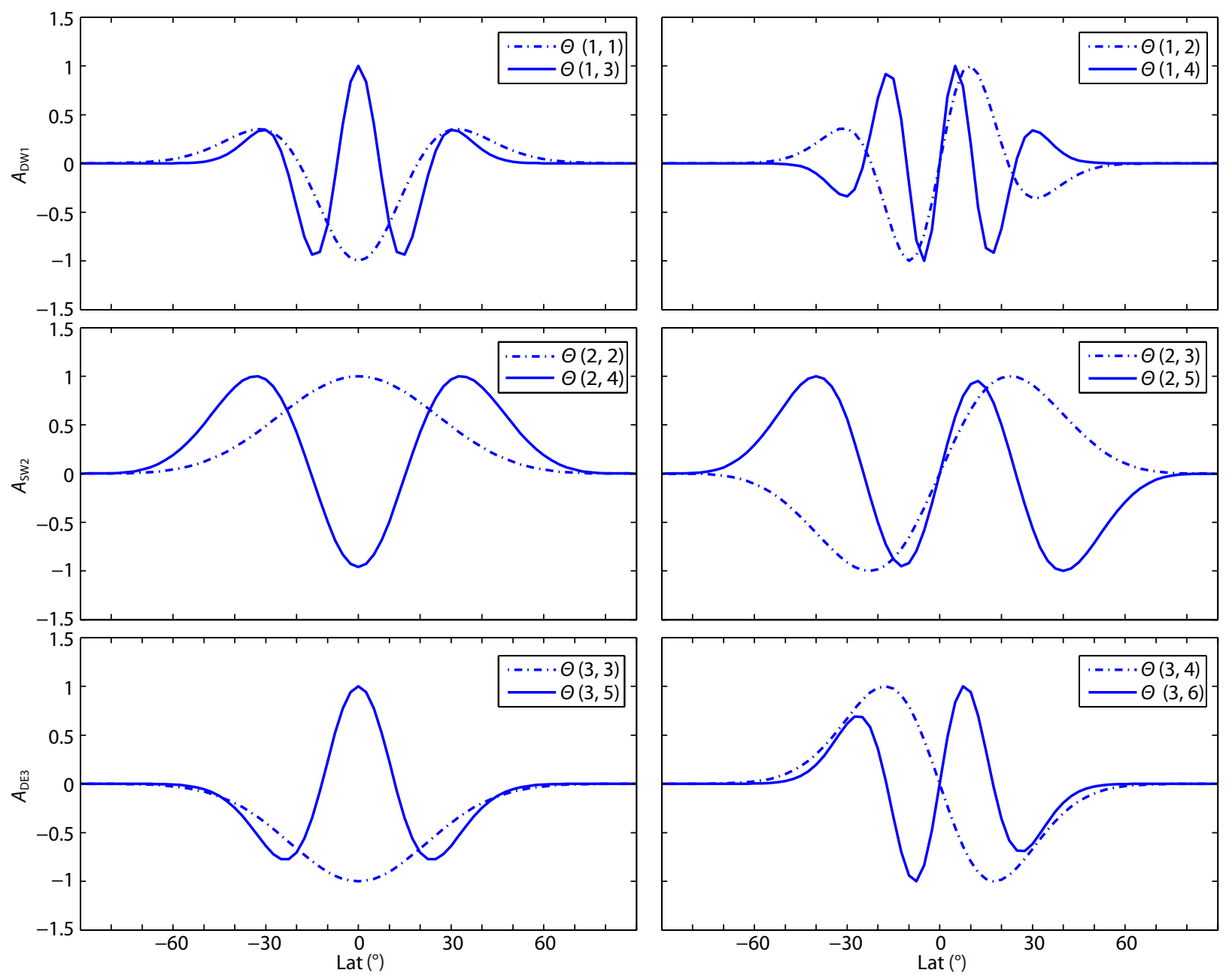

Figure 1. Normalized propagating Hough modes as a function of latitude for the DW1, SW2, and DE3 tides, respectively. The Hough modes in the left column are symmetric about the equator, and those in the right column are antisymmetric. 
Table 1. Lamb numbers (eigenvalues) of the Hough modes (in km).

\begin{tabular}{ccccccc}
\hline \multicolumn{7}{c}{ Hough mode } \\
\hline$(s, n)$ & $(1,1)$ & $(1,3)$ & $(2,2)$ & $(2,4)$ & $(3,3)$ & $(3,5)$ \\
$\varepsilon_{n}^{s}$ & 0.6940 & 0.1208 & 7.8647 & 2.1176 & 2.2461 & 0.3332 \\
$(s, n)$ & $(1,2)$ & $(1,4)$ & $(2,3)$ & $(2,5)$ & $(3,4)$ & $(3,6)$ \\
$\varepsilon_{n}^{s}$ & 0.2395 & 0.0728 & 3.6770 & 1.3730 & 0.7824 & 0.1700 \\
\hline
\end{tabular}

density, and zonal wind, respectively. We used this expression to calculate the Hough modes of the atmospheric tidal components and the sources of the tides at each altitude. The derivation process is described in detail in the Appendix.

\section{Results}

\subsection{Sources of the Tidal Components}

Figure $2 \mathrm{a}$ and $2 \mathrm{~b}$ show, respectively, the contours of the amplitudes $A$ and the phases $t_{0}$ of the yearly mean main tidal sources as they are distributed in the plane of height versus latitude. From left to right, the plots refer the three tidal sources: DW1, SW2, and DE3. In addition, the high-latitude tidal source properties were not included in this work, and Figure 2 gives only the amplitudes and phases of the sources at the low and middle latitudes (within $\left.\pm 60^{\circ}\right)$. Figure 2 clearly illustrates that the DE3 source is the strongest and that it is strongest at $5-10 \mathrm{~km}$.

To research the yearly variations of the sources in detail, both the amplitudes and the phases of the three main tidal sources at $6 \mathrm{~km}$ were further averaged across different years (1988-2011). These results are displayed in Figure 3 as distributions of latitudes and months.

\subsubsection{DW1}

As shown in the left column plot of Figure $2 \mathrm{a}$ and the top left plot of Figure 3, the DW1 source presents mainly in the lower latitudes at $\pm 10^{\circ}$ latitude, and in the northern hemisphere, it is stronger than in the southern hemisphere. The DW1 source occurs mainly in the altitudes from 5 to $8 \mathrm{~km}$, and the peak is presented at $6 \mathrm{~km}$, as illustrated in the left plot of Figure 2a. As shown in the top left plot of Figure 3, the DW1 source behaves as a weak seasonal variation in the lower latitudes (about $\pm 10^{\circ}-15^{\circ}$ ). In other words, the amplitudes of the DW1 source are larger in the summer months than in the winter months, and the DW1 source presents semi-annual variation near the equator $\left( \pm 10^{\circ}\right)$ such that it is larger at the equinoxes than at the solstices. For instance, during the summer months (i.e., July, August, and September) in the northern hemisphere, the amplitudes at $10^{\circ}$ latitude may reach to about $300 \mathrm{~J}$, whereas they drop to $50 \mathrm{~J}$ during the winter months. Along the equator, the DW1 source may be close to $320 \mathrm{~J}$ during the equinoxes, whereas it may be only $100 \mathrm{~J}$ during the solstices.

The phases of the DW1 source are illustrated in the left plot of Figure $2 \mathrm{~b}$ as well as the bottom left plot of Figure 3 . The figure shows that the phases of the DW1 source are stable with latitude and change regularly with the months. A weak seasonal variation is also shown, in which the crests of the equatorial source appear $\sim 9$ hours earlier in the summer than in the winter and $\sim 12$ hours at the middle latitudes, as shown in the bottom left plot of Figure 3.

\subsubsection{SW2}

As shown in the centre plot of Figure $2 a$ and $2 b$ and the top centre plot of Figure 3, the SW2 source appears mainly at the middle latitudes $\left( \pm 30^{\circ}\right)$. It should be noted that the SW2 source is symmetric relative to the equator and that the source in the southern hemisphere is a little stronger than in the northern hemisphere. As shown in the centre plot of Figure $2 a$ and $2 b$, the SW2 source mainly presents at altitudes of $5-10 \mathrm{~km}$, and the peak is at $7 \mathrm{~km}$. As displayed in the top centre plot of Figure 3, the SW2 source presents a remarkable annual and semi-annual variation in which the amplitudes are largest during the March equinox months and larger during the June solstice months. For example, in March, the amplitudes at $\pm 30^{\circ}$ latitude may reach to about $250 \mathrm{~J}$, whereas in June, the amplitudes at $-30^{\circ}$ drop to $180 \mathrm{~J}$ and at $30^{\circ}$ latitude, they drop to $100 \mathrm{~J}$.

As shown in the centre plot of Figure $2 \mathrm{~b}$ and the bottom centre plot of Figure 3, the phases of the SW2 source present a seasonal variation. Note that the crests of the source during the summer months are about 6 hours earlier than those in the winter months.

\subsubsection{DE3}

The DE3 source appears mainly around the equatorial latitudes within about $\pm 30^{\circ}$, and it is stronger in the southern hemisphere than in the northern hemisphere, as shown in the right plot of Figure $2 \mathrm{a}$ and the top right plot of Figure 3. This source presents an obvious semi-annual variation such that the amplitudes in the southern hemisphere are larger in the months around solstices than in the months around equinoxes. In contrast, the DE3 source in the northern hemisphere is larger in the months around equinoxes than in the months around solstices, as shown in the top right plot of Figure 3. Particularly in January, the amplitudes at $-10^{\circ}$ latitude may be $500 \mathrm{~J}$, and the amplitudes in March drop to half those at the low latitudes.

As shown in the right plot of Figure $2 \mathrm{~b}$ and the bottom right plot of Figure 3, the phases of the DE3 source are stable across the months and manifest as a weak seasonal variation that crests during the summer months $\sim 8$ hours later than during the winter months. That is, in the northern hemisphere, the crests occur in the evening during the summer months and in the morning during the winter months.

\subsection{Modes of the Tidal Components}

On the basis of the HMD, we describe the height profile of the nine leading modes $\left(C_{n}\right)$ of DW1, SW2, and DE3 in Figure 4. The modes of the tidal components present the most obvious convergence tendency: the amplitudes of the modes decrease as the series numbers increase. Specifically, the DW1 component is dominated by the first symmetric $(1,1)$ mode, whereas for the SW2 components, the corresponding dominant modes are the asymmetric modes $(2,3)$, and substantial contributions of the DE3 components are also from the symmetric modes $(3,3)$. Hence, we suggest that the main features of the tidal components are captured by the three leading modes, as shown in Figure 4 . We therefore confined our investigation to the three leading Hough modes of 


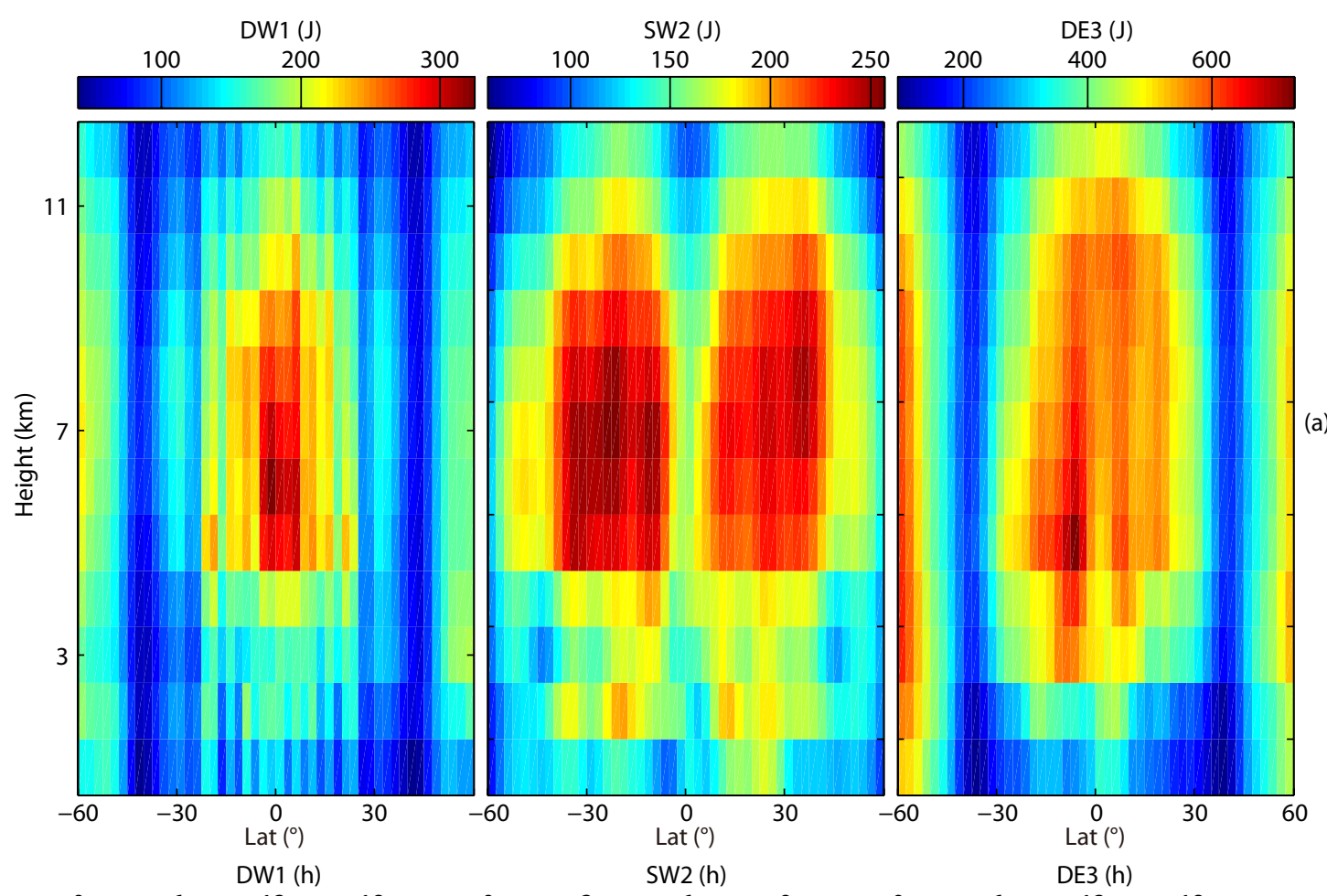

(a)
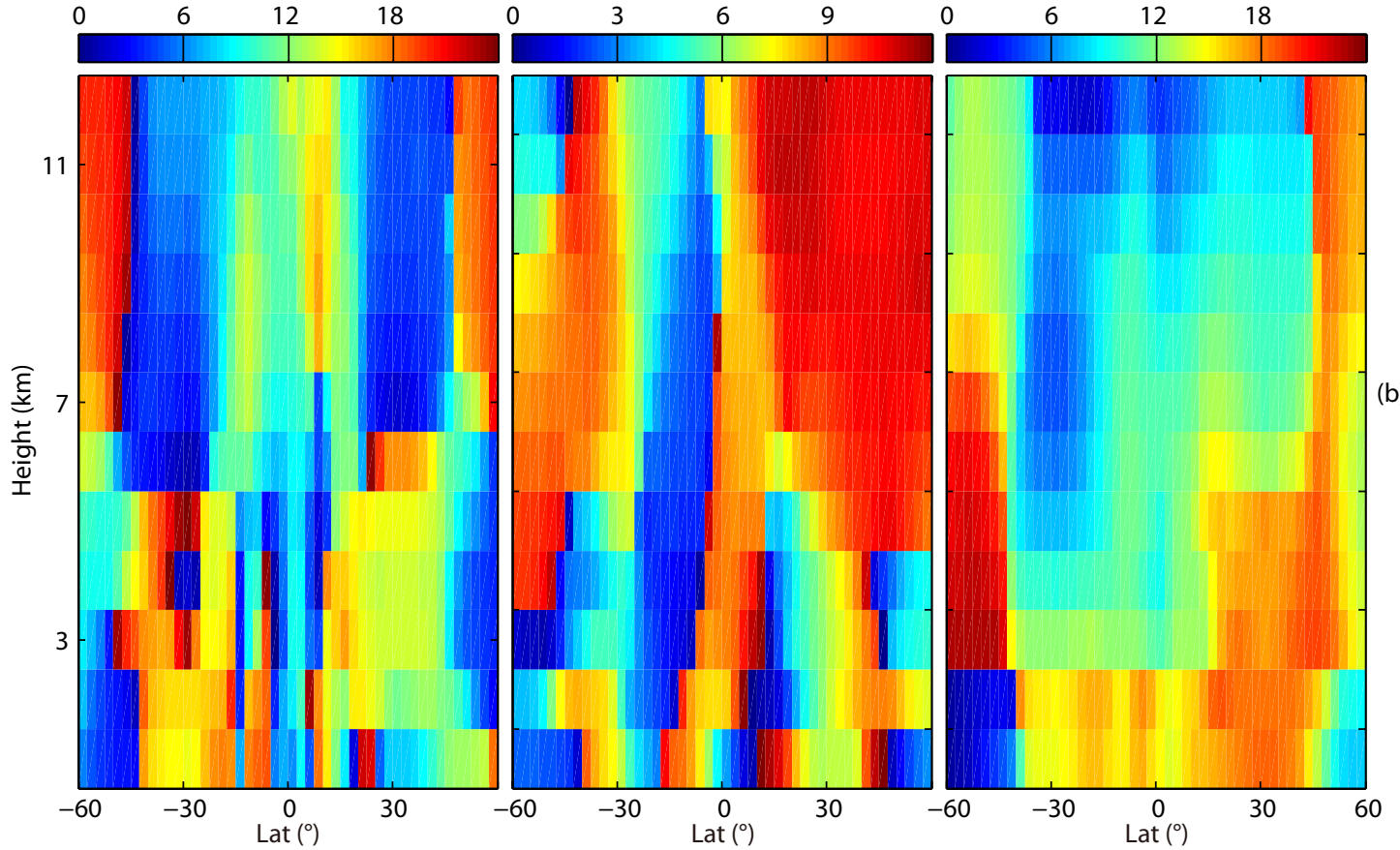

Figure 2. (a) Height profiles of the main tidal sources, from left to right, for DW1, SW2, and DE3. The data are the amplitudes $A$ of the mean tides from 1998 to 2011. (b) Same as Figure 2a but for the phases $t_{0}$ of the main tidal sources.

the tidal components in the rest of this study.

Figure $5 \mathrm{a}$ and $5 \mathrm{~b}$ illustrate the amplitudes $A$ and phases $t_{0}$ of the Hough mode coefficients of the main tidal components at $6 \mathrm{~km}$, respectively. The different Hough modes are denoted by the following solid lines: blue, red, and green.

\subsubsection{DW1}

As shown in the top left plot of Figure $5 a$, for DW1, the amplitudes of the first symmetry mode $(1,1)$ are largest, and they are larger after the year 2000 than before. Furthermore, the $(1,1)$ mode has an obvious inter-annual variation; that is, the source amplitudes are larger in February, July, and November than in the other months, as shown in the top right plot of Figure $5 \mathrm{a}$. The first asymmetry mode $(1,2)$ manifests as an obvious semi-annual variation that is larger in April and November. The third mode $(1,3)$ presents a weak annual and semi-annual variation that is larger during the solstices than the equinoxes and is largest during the June solstices. 

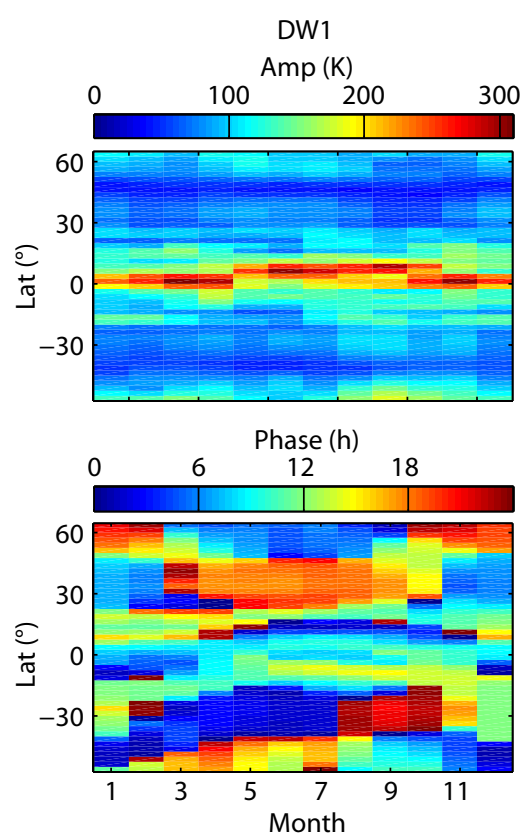

SW2

Amp (K)
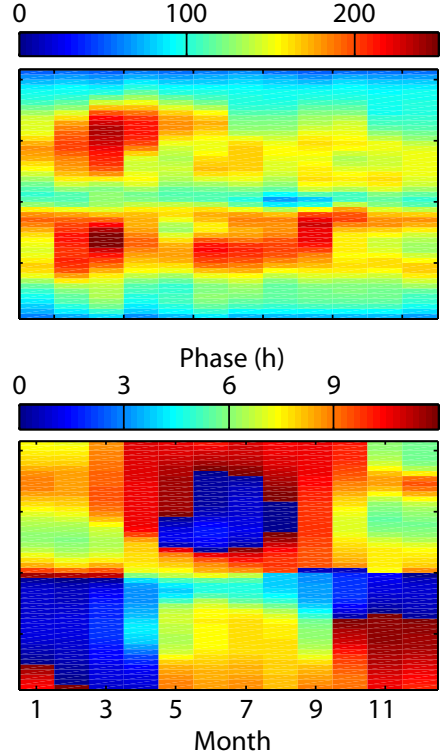
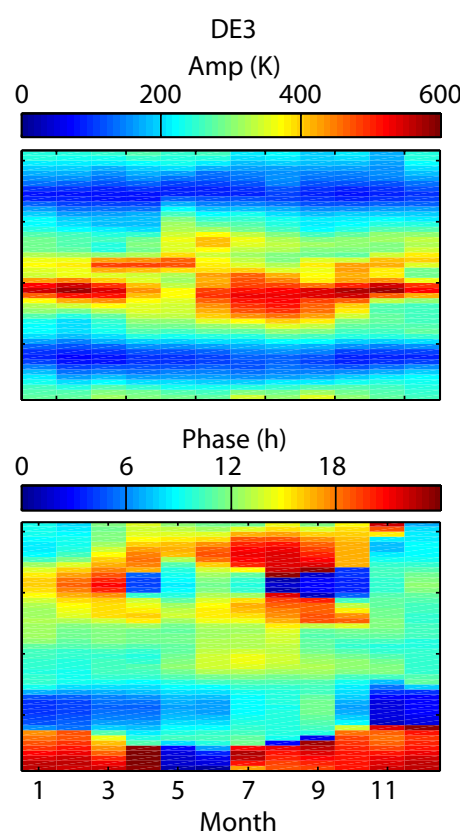

Figure 3. Distributions of the monthly mean tidal sources of DW1 (left column), SW2 (center column), and DE3 (right column). The top plots are the amplitudes $A$ and the bottom plots are the phases $t_{0}$. The monthly mean sources are statistically averaged among different years from 1988 to 2011.
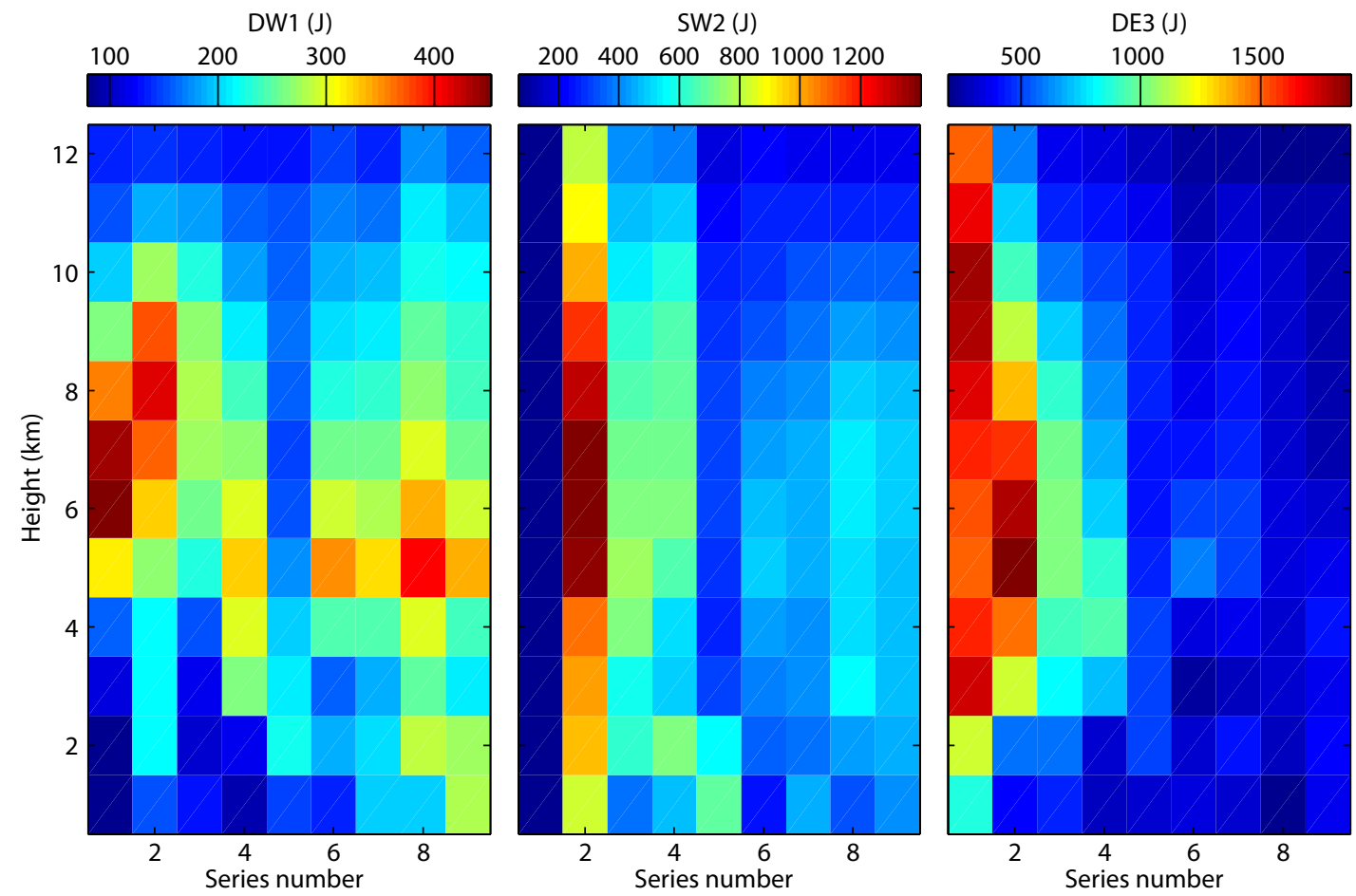

Figure 4. Amplitudes of the nine leading Hough mode coefficients $C_{n}$. The left plot is DW1, the center is SW2, and the right is DE3.

The phases of the Hough modes for DW1 are illustrated in the top plots of Figure $5 \mathrm{~b}$. As shown in the figure, the $(1,1)$ and $(1,3)$ modes are very stable before 2000 , and the crests are gradually delayed by one hour after 2000 . The $(1,1)$ mode displays an obvious yearly variation: the $(1,1)$ mode crests during the June solstice after $\sim 7$ hour universal time (UT) compared with that during the December solstice.

\subsubsection{SW2}

The amplitudes of the symmetry mode $(2,2)$ are very stable and do not display obvious yearly variations, as shown in the middle plots of Figure $5 \mathrm{a}$. In addition, the amplitudes of the $(2,2)$ mode are smaller than those of the $(2,3)$ and $(2,4)$ modes. In contrast, the amplitudes of the $(2,3)$ mode do not present an obvious interannual variation, and they occur as a semi-annual variation that is 

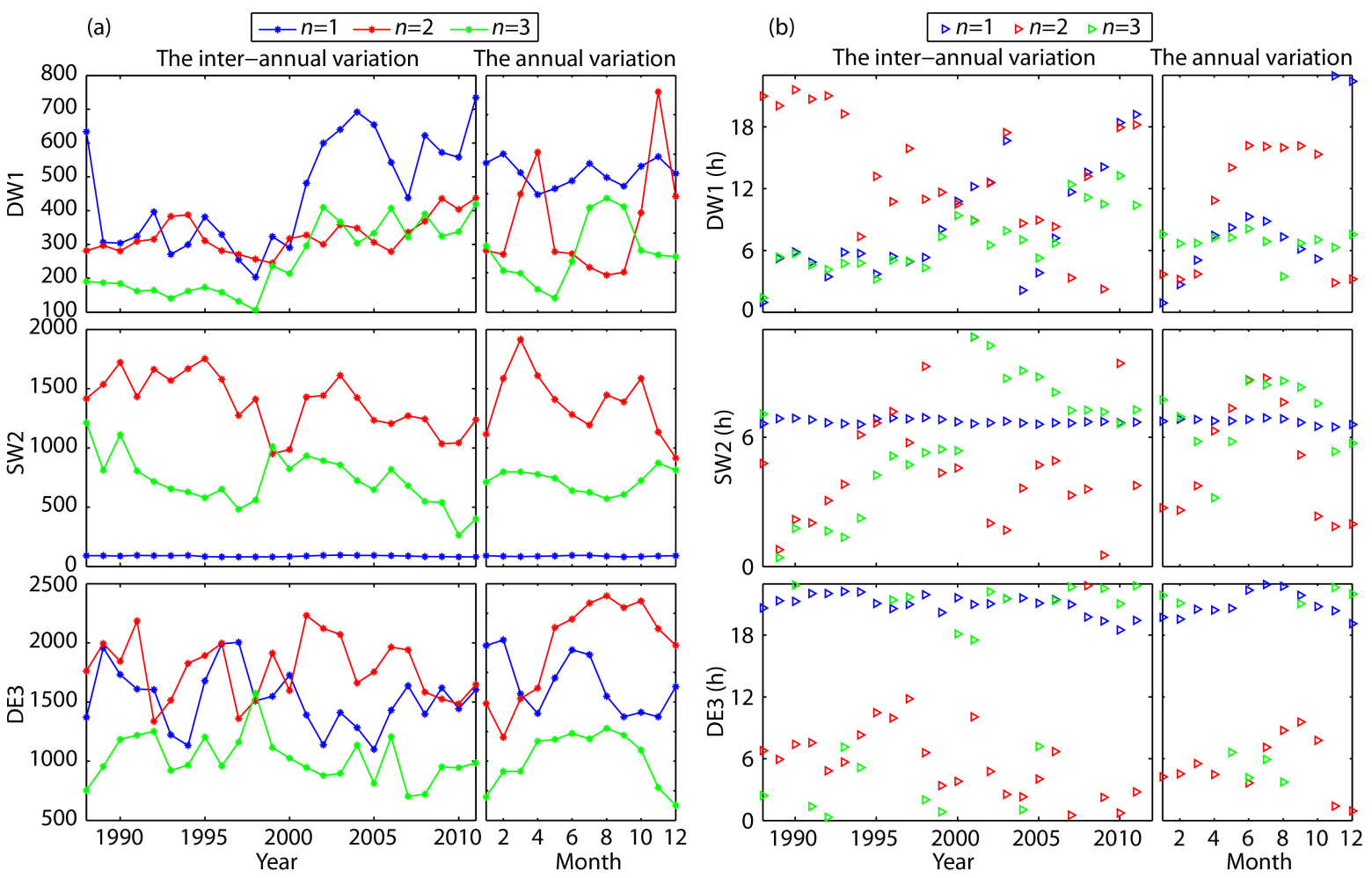

Figure 5. (a) Amplitudes $A$ of the three leading Hough mode coefficients $C_{n}$ of the main tidal component. The top plots are DW1, the middle plots are SW2, and the bottom plots are DE3. The left column is the interannual variation, and the right column is the annual variation. (b) Same as (a) but for the phases $t_{0}$ of the main tidal components.

larger during the equinoxes than the solstices, as illustrated in the middle left plot in Figure 5 a. Similar to the $(2,2)$ mode, the $(2,4)$ mode is stable and presents a weak semi-annual variation that is larger during the equinox than the solstices, as displayed in the middle right plot of Figure $5 \mathrm{~b}$.

The middle plot of Figure $5 \mathrm{~b}$ shows the phases $t_{0}$ of the SW2 modes at $6 \mathrm{~km}$. Similarly, the phases of the $(2,2)$ mode are very stable and do not manifest a yearly variation; that is, the $(2,2)$ mode crests in $\sim 7$ hour. The phases of the $(2,3)$ mode are unstable and occur as an annual variation that crests during the June solstice in $\sim 8$ hour, after that during the December solstice. The phases of the $(2,4)$ mode are gradually delayed from 1988 to 2011.

\subsubsection{DE3}

The first mode $(3,3)$ and the second mode $(3,4)$ are obviously strong at $6 \mathrm{~km}$, as illustrated in the bottom plots of Figure $5 \mathrm{~b}$. The annual variation of the $(3,3)$ mode is larger in February and June, as shown in the bottom right plot of Figure $5 \mathrm{a}$. Even though the $(3,4)$ mode has an indistinct cycle of 5 years, it is larger in the second half of the year than in the first half. The $(3,5)$ mode is smaller and its yearly variation is not obvious, as illustrated in the bottom plots of Figure 5 a.

The bottom plots of Figure $5 \mathrm{~b}$ show the phases $t_{0}$ of the DE3 Hough coefficients at $6 \mathrm{~km}$. The phases of the $(3,3)$ and $(3,5)$ modes are very stable and do not manifest as yearly variations, that is, the $(3,3)$ mode crests in the evening and the $(3,4)$ mode crests in the evening and before dawn. The phases of the $(3,4)$ mode are unstable, and this mode crests in the morning, as shown in the bottom plots of Figure $5 \mathrm{~b}$.

\section{Discussion}

The ENSO phenomenon is the most prominent source of climate variability. Previous studies have indicated that coherent temperature signals are evidence that the ENSO modulates upwelling in the tropical lower stratosphere (Manzini, 2009). Large-scale changes in precipitation and surface temperatures in the tropical lower stratosphere are known to be related to the ENSO phenomenon (e.g., see Vial et al., 1994; Trenberth et al., 2002; Lieberman et al., 2007; Randel et al, 2009).

The long-term and altitudinal variability of the sources of the three tidal components (i.e., DW1, SW2, and DE3, derived by HMD) at corresponding latitudes are displayed in Figure 6. To show the main variation of the sources, the DW1 and DE3 sources in the top and bottom plots of Figure 6 are the average of the low latitudes $\left( \pm 30^{\circ}\right)$, and the SW2 sources in the middle plot are the average of the low to middle latitudes $\left( \pm 60^{\circ}\right)$. To research the relationship between these results and the ENSO, we compared the atmospheric tidal sources retrieved from the CFSR with those predicted by the Multivariate ENSO Index (MEI) from the National Oceanic and Atmospheric Administration Physical Sciences Laboratory, downloaded from the website http://www.esrl.noaa.gov/ psd/enso/mei/. In each plot of Figure 6, the white lines represent the long-term MEI.

Figure 6 shows that the diurnal migrating (DW1) tidal source is coupled with the MEI and that the peak source presents at a high- 


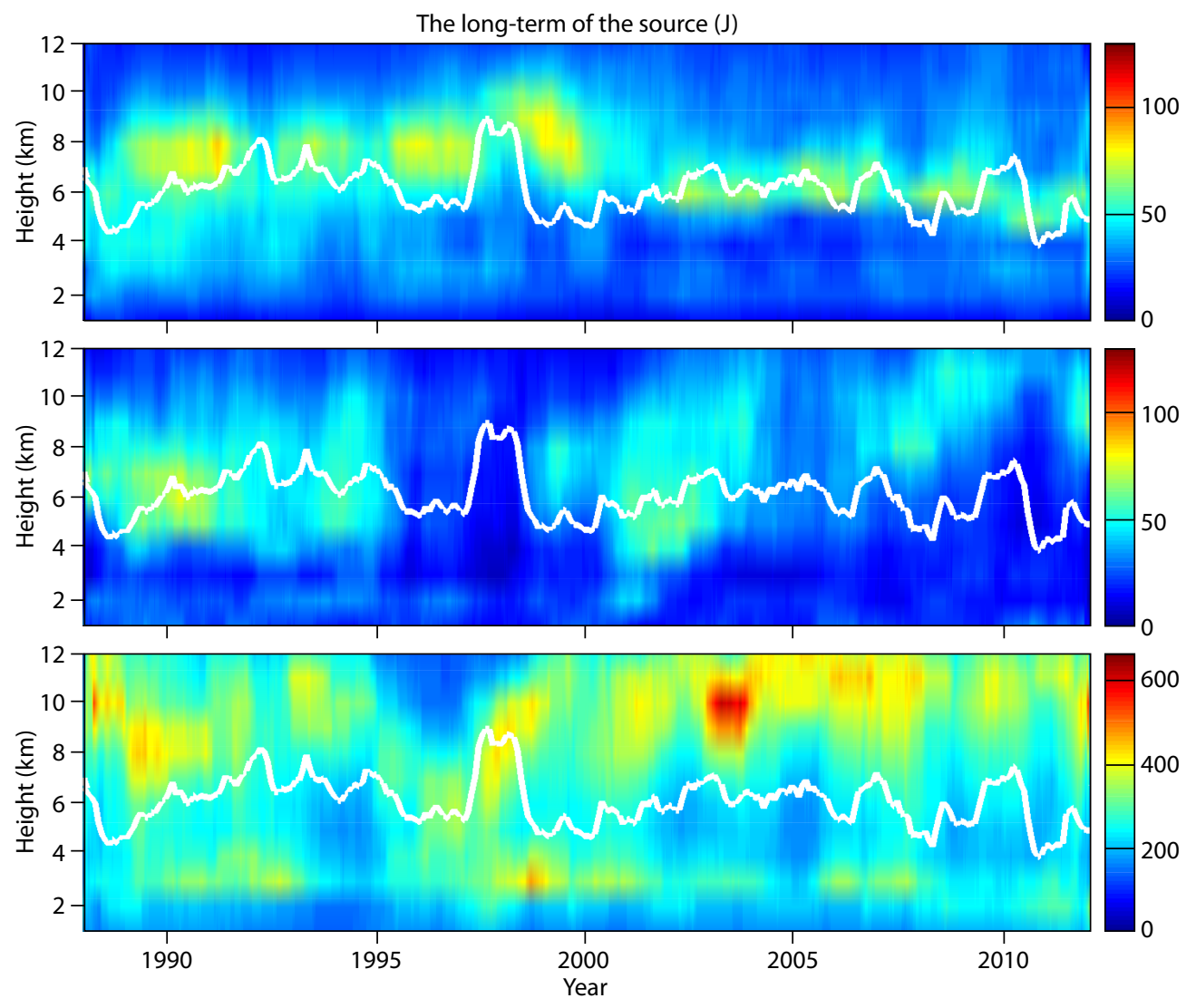

Figure 6. Long-term variability of the sources. The top plot is DW1, the middle plot is SW2, and the bottom plot is DE3. In each plot, the white lines represent the long-term Multivariate ENSO Index (MEI).

er altitude when the ENSO is stronger. As illustrated in the middle plot of Figure 6, the SW2 source is different from the DW1 source in that it is weak when the ENSO is strong, especially from 1997 to 1998, when the SW2 source almost disappears. Regarding the DE3 source, the ENSO phenomenon hardly affects it, as shown in the bottom plot of Figure 6 . The result above approximately agrees with earlier research. For example, Gurubaran et al. (2005) observed a decrease in the amplitude of the diurnal tide at mesopause altitudes and attributed this to an increase in the strength of nonmigrating tides associated with the ENSO. Lieberman et al. (2007) reported that the heating of water vapor contributed to the tidal enhancement observed from 1997 to 1998 and that the altered heating patterns resulted in a stronger forcing of the migrating diurnal tide (DW1) by the heated water vapor. In addition, our result above agrees with the results of Lieberman et al. (2007).

\section{Summary and Conclusions}

In this work, we analyzed the inter-annual and yearly variations of the tropospheric tidal sources and Hough coefficients (e.g., DW1, SW2, and DE3) from the NCEP CFSR data from 1988 to 2011. The main results are as follows:

The diurnal sources (i.e., DW1 and DE3) are stronger around the equator at $5-10 \mathrm{~km}$. The semi-diurnal source SW2 is stronger in the latitude range around $\pm 30^{\circ}$ at $5-10 \mathrm{~km}$, and it is symmetric about the equator. The SW2 source is stronger in the southern hemisphere than in the northern hemisphere.
(1) The DW1 source behaves with a weak seasonal variation in the lower latitudes (about $\pm 10^{\circ}-15^{\circ}$ ) such that the amplitudes of the DW1 source are larger in summer than in winter. Near the equator, the DW1 source presents semi-annual variation that is stronger during the equinoxes than the solstices. Moreover, the first Hough mode of the DW1 component, the $(1,1)$ mode, dominates the DW1 tidal components in the higher troposphere.

(2) The SW2 source remarkably manifests the symmetry of the southern and northern hemisphere, and it is stronger in the southern hemisphere than in the northern hemisphere. The SW2 source presents an obvious annual and semi-annual variation such that the amplitudes are largest in March, larger in June, and smallest in other months. Furthermore, the strongest mode is the second mode $(2,3)$, and it presents a remarkable semi-annual variation that is stronger at the equinoxes than the solstices. Moreover, the symmetric Hough modes of the SW2 component, the $(2,2)$ and $(2,4)$ modes, are weak and present slight yearly variations.

(3) The DE3 source mainly occurs around the equatorial latitudes and presents an obvious semi-annual variation. In the southern hemisphere, the amplitudes are larger in the months around the solstices than in the months around the equinoxes, although in the northern hemisphere, the source amplitudes are slightly larger at the equinoxes than the solstices. Like the DW1 component, the first Hough mode of the DE3 component $(3,3)$ is the main mode, and it shows an obvious annual variation that is strongest 
during the June solstice.

(4) These results show a strong ENSO signal in the migrating tidal source (i.e., DW1 and SW2) in the higher troposphere from the CFSR set. There is good agreement between the time series of ENSO signals (MEI) and the long-term variability of the diurnal source amplitudes, whereas there is disagreement between the ENSO and the semidiurnal source amplitudes.

The implications of the results presented in this work can be easily recognized, considering that the atmospheric tides carry heat energy from the troposphere into the middle atmosphere and the mesosphere and lower thermosphere region. We thus suggest that an HMD analysis of the tidal components can be used when studying and modelling atmospheric tides.

\section{Appendix A}

In this section, we briefly show the derivation process of the HMD of the atmospheric tidal components and the sources of the tides at each altitude. The mixture of gases in the lower and middle atmosphere can be treated as a single ideal gas of constant with molecular weight $M$. The basic hydrodynamic and thermodynamic laws of this atmospheric gas may be represented, for example, by Kato (1980).

The equation of motion is

$$
\frac{\mathrm{d} \boldsymbol{V}}{\mathrm{d} t}=-\frac{1}{\rho} \nabla p-2 \boldsymbol{\Omega} \times \boldsymbol{V}+\boldsymbol{g}+\boldsymbol{F}
$$

the equation of mass continuity is

$$
\frac{\mathrm{d} \ln \rho}{\mathrm{d} t}+\nabla \cdot \boldsymbol{V}=0
$$

the first law of thermodynamics is

$$
C_{p} \frac{\mathrm{d} T}{\mathrm{~d} t}-\frac{1}{\rho} \frac{\mathrm{d} P}{\mathrm{~d} t}=\dot{Q}
$$

and the ideal gas equation is

$$
P=\rho R T
$$

where $t$ is time; $\mathrm{d} / \mathrm{d} t=\partial / \partial t+(v \cdot \nabla)$ is the total time derivative; $\boldsymbol{V}$ is the velocity vector with its components directed to the east $u$, to the north $v$, and upward $w ; \Omega$ is the vector of the earth's rotation $\left(\Omega=7.29 \times 10^{-5} \mathrm{~s}^{-1}\right) ; \boldsymbol{g}$ is the apparent acceleration of the Earth's gravity $\left(g=9.81 \mathrm{~m} / \mathrm{s}^{2}\right) ; P$ is pressure; $\rho$ is density; $T$ is temperature; $\boldsymbol{F}$ is the momentum force per unit mass (e.g., lunar tidal force or frictional force); $C_{p}$ is the specific heat at constant pressure; $\dot{Q}$ is the net heat input per unit mass or net heat flux divergence; and $R$ is the gas constant $\left(R=287 \mathrm{~J} \cdot \mathrm{kg}^{-1} \cdot K^{-1}\right)$.

For convenience in later applications, we have separated the total temperature $T$, the total pressure $P$, the total density $\rho$, the total heat $\dot{Q}$, and the total momentum force $\boldsymbol{F}$ into a global mean (or a basic state) depending only on height $z$ (e.g., $T(z))$ and departures from that global mean (e.g., $T(z, \phi, \lambda, t)$ ), which depend additionally on altitude $z$, latitude $\phi$, longitude $\lambda$, and time $t$. As we know, it is sufficient to study periodic waves of angular frequency $v$ and decompose the waves into their modal structures, zonal wavenumber $m$. This means that the equations of motion can be solved by separation of the variables $(\phi, \lambda, t$, and $z)$ and that the modal structure becomes an eigenvalue problem. The eigenvalues of the individual modes and their horizontal structures had been represented, for example, by Volland (1988). In this section, we present the vertical modal structures.

First, as reported by Kato (1980), we assume the following atmospheric parameters:

$$
\left\{\begin{array}{l}
\hat{X}=\hat{X}_{1}(z, v) \theta_{n}^{m}(\phi, w) \exp [\mathrm{i}(m \lambda-v t)], \\
w=W_{1}(z, v) \theta_{n}^{m}(\phi, w) \exp [\mathrm{i}(m \lambda-v t)], \\
p=P_{1}(z, v) \theta_{n}^{m}(\phi, w) \exp [\mathrm{i}(m \lambda-v t)], \\
\rho=\rho_{1}(z, v) \theta_{n}^{m}(\phi, w) \exp [\mathrm{i}(m \lambda-v t)], \\
\dot{Q}=\dot{Q}_{1}(z, v) \theta_{n}^{m}(\phi, w) \exp [\mathrm{i}(m \lambda-v t)],
\end{array}\right.
$$

where

$$
\hat{X}=\nabla \cdot \boldsymbol{U}+\frac{\partial w}{\partial z}-\frac{\dot{Q}}{C_{p} T_{0}}
$$

With reference to Kato (1980), we can obtain the following equation:

$$
\frac{\partial^{2} \hat{X}_{1}}{\partial z^{2}}-\frac{1}{H} \frac{\partial \hat{X}_{1}}{\partial z}+\frac{g \varepsilon}{4 a^{2} \Omega^{2}} \cdot \frac{(\gamma-1)}{\gamma H} \hat{X}_{1}=-\frac{g \varepsilon}{4 a^{2} \Omega^{2}} \cdot \frac{1}{\gamma H} \cdot \frac{\dot{Q}}{C_{p} T_{0}}
$$

where

$$
H=\frac{R T_{0}}{g}, \gamma=\frac{C_{p}}{C_{v}}
$$

and $\varepsilon$ is a separation constant known as the Lamb parameter. This parameter is the eigenvalue of the modal structure.

We can obtain

$$
\hat{X}_{1}=C \exp \left[\frac{z}{2 H}(1-k)\right]-\frac{1}{\gamma-1} \cdot \frac{\dot{Q}_{1}}{C_{p} T_{0}},
$$

where $C=\Omega, k=\operatorname{sign}(\omega) \times \sqrt{1-\frac{(\gamma-1) g H \varepsilon_{n}^{m}}{\gamma a^{2} \Omega^{2}}}$.

Because of $\frac{\partial \hat{X}_{1}}{\partial z}=\left(\hat{X}_{1}+\frac{1}{\gamma-1} \cdot \frac{\dot{Q}_{1}}{C_{p} T_{0}}\right) \cdot \frac{1-k}{2 H}$,

$$
\hat{X}_{1}=\frac{2 H}{1-k} \cdot \frac{\partial \hat{X}_{1}}{\partial z}-\frac{1}{\gamma-1} \cdot \frac{\dot{Q}_{1}}{C_{p} T_{0}} \text {. }
$$

From (A1) to (A4), we can obtain

$$
\left\{\begin{array}{l}
\frac{\partial W_{1}}{\partial z}=(1-\gamma) \hat{X}_{1}+\frac{\gamma R T_{0}}{g} \frac{\partial \hat{X}_{1}}{\partial z}+\frac{\dot{Q}}{C_{p} T_{0}} \\
\frac{\partial P_{1}}{\partial t}=-\frac{\partial P_{0}}{\partial z} W_{1}-\gamma \rho_{0} R T_{0} \hat{X}_{1} \\
\rho_{1}=-\frac{1}{g} \frac{\partial P_{1}}{\partial z} \\
T_{1}=T_{0}\left(\frac{P_{1}}{P_{0}}-\frac{\rho_{1}}{\rho_{0}}\right)
\end{array}\right.
$$

We can construct an auxiliary equation as follows:

$$
\nabla \cdot \boldsymbol{U}=-\nabla^{2} \xi=-\frac{\varepsilon}{4 a^{2} \Omega^{2}} \cdot \frac{\partial \Phi}{\partial t}
$$

where $\Phi$ is the local geopotential.

By introducing (A10) into (A11),

$$
\frac{\partial W_{1}}{\partial z}=\left[\frac{2(1-\gamma)}{1-k} H+\gamma H\right] \frac{\partial \hat{X}_{1}}{\partial z}+\frac{2 \dot{Q}_{1}}{C_{p} T_{0}} .
$$


We assume that $\frac{2 \dot{Q}_{1}}{C_{p} T_{0}}=\frac{\partial J_{1}}{\partial z}$ and introduce it into (A16),

$$
\frac{\partial W_{1}}{\partial z}=\left[\frac{2(1-\gamma)}{1-k} H+\gamma H\right] \frac{\partial \hat{X}_{1}}{\partial z}+\frac{\partial J_{1}}{\partial z}
$$

We can obtain that

$$
W_{1}=\left[\frac{2(1-\gamma)}{1-k} H+\gamma H\right] \hat{X}_{1}+J_{1}
$$

In this equation, we suggest that $J_{1}$ is the source of the atmospheric tides.

By introducing (A18) into (A12) to (A14), we can obtain

$$
\left\{\begin{array}{l}
W_{1}=\left[\frac{2(1-\gamma)}{1-k} H+\gamma H\right] \hat{X}_{1}+J_{1}, \\
\frac{P_{1}}{P_{0}}=\mathrm{i} \cdot \frac{2(1-\gamma)}{v(1-k)} \exp \left[\frac{z}{2 H}(1-k)\right]+\mathrm{i} \cdot \frac{2}{v \Omega(1-k)} \cdot \frac{\dot{Q}_{1}}{C_{p} T_{0}}+\mathrm{i} \cdot \frac{J_{1}}{v \Omega H^{\prime}} \\
\frac{\rho_{1}}{\rho_{0}}=\mathrm{i} \cdot \frac{(1-\gamma)(1+k)}{v(1-k)} \exp \left[\frac{z}{2 H}(1-k)\right]-\mathrm{i} \cdot \frac{2 k}{v \Omega(1-k)} \cdot \frac{\dot{Q}_{1}}{C_{p} T_{0}}+\mathrm{i} \cdot \frac{J_{1}}{v \Omega H^{\prime}} \\
\frac{T_{1}}{T_{0}}=\mathrm{i} \cdot \frac{(1-\gamma)}{v} \exp \left[\frac{z}{2 H}(1-k)\right]+\mathrm{i} \cdot \frac{2 \dot{Q}_{1}}{v \Omega C_{p} T_{0}},
\end{array}\right.
$$

where

$$
\frac{2 \dot{Q}_{1}}{C_{p} T_{0}}=\frac{\partial J_{1}}{\partial z}
$$

From the analysis of the above, we can obtain

$$
\frac{P_{1}}{\rho_{0}}=\Phi_{n}^{m}(z, \omega)
$$

Therefore,

$$
\Phi_{n}^{m}=\frac{P_{1}}{\rho_{0}}=\mathrm{i} \cdot \frac{2 R T(1-\gamma)}{v(1-k)} \exp \left[\frac{z}{2 H}(1-k)\right]+\mathrm{i} \cdot \frac{2 H g}{v \Omega(1-k)} \cdot \frac{\dot{Q}_{1}}{C_{p} T_{0}}+\mathrm{i} \cdot \frac{g J_{1}}{v \Omega} .
$$

Because of (A13),

$$
U_{n}^{m}=\frac{\sqrt{\varepsilon_{n}^{m}}}{2 a \Omega} \Phi_{n}^{m}=\mathrm{i} \cdot \frac{R T(1-\gamma) \sqrt{\varepsilon_{n}^{m}}}{a \Omega v(1-k)} \exp \left[\frac{z}{2 H}(1-k)\right]+\mathrm{i} \cdot \frac{H g \sqrt{\varepsilon_{n}^{m}}}{a \Omega^{2} v(1-k)} \cdot \frac{\dot{Q}_{1}}{C_{p} T_{0}}+\mathrm{i} \cdot \frac{g J_{1} \sqrt{\varepsilon_{n}^{m}}}{2 a v \Omega^{2}} .
$$

Hence, we can decompose temperature, density, and zonal wind with the above equations. We can obtain

$$
\left\{\begin{array}{l}
\rho=C_{n}^{m} \cdot\left\{\frac{\mathrm{i}(1-\gamma)(1+k)}{v(1-k)} \exp \left[\frac{z}{2 H}(1-k)\right]-\mathrm{i} \cdot \frac{2 k}{v \Omega(1-k)} \cdot \frac{\dot{Q}_{1}}{C_{p} T_{0}}+\mathrm{i} \cdot \frac{J_{1}}{v \Omega H}\right\} \rho_{0} \cdot \Theta_{n}^{m}, \\
T=C_{n}^{m} \cdot\left\{\mathrm{i} \cdot \frac{(1-\gamma)}{v} \exp \left[\frac{z}{2 H}(1-k)\right]+\mathrm{i} \cdot \frac{2 \dot{Q}_{1}}{v \Omega C_{p} T_{0}}\right\} \cdot \Theta_{n}^{m}, \\
U=C_{n}^{m} \cdot\left\{\mathrm{i} \cdot \frac{R T(1-\gamma) \sqrt{\varepsilon_{n}^{m}}}{a \Omega v(1-k)} \exp \left[\frac{z}{2 H}(1-k)\right]+\mathrm{i} \cdot \frac{H g \sqrt{\varepsilon_{n}^{m}}}{a \Omega^{2} v(1-k)} \cdot \frac{\dot{Q}_{1}}{C_{p} T_{0}}+\mathrm{i} \cdot \frac{g J_{1} \sqrt{\varepsilon_{n}^{m}}}{2 a v \Omega^{2}}\right\} \cdot \Theta_{n}^{m},
\end{array} .\right.
$$

and

$$
C_{n}^{m} \cdot\left[\begin{array}{ccc}
P \cdot \rho_{z} \cdot \rho_{0} & \mathrm{i} \cdot \frac{1}{v \Omega H} \cdot \rho_{0} & \mathrm{i} \cdot \frac{1}{v \Omega C_{p} T_{0}} \cdot \frac{2 k}{(1-k)} \cdot \rho_{0} \\
P \cdot T_{z} \cdot T_{0} & 0 & \mathrm{i} \cdot \frac{2}{v \Omega C_{p} T_{0}} \cdot T_{0} \\
P \cdot U_{z} & \mathrm{i} \cdot \frac{g \sqrt{\varepsilon_{n}^{m}}}{2 a v \Omega^{2}} & \mathrm{i} \cdot \frac{1}{v \Omega C_{p} T_{0}} \cdot \frac{H g \sqrt{\varepsilon_{n}^{m}}}{a \Omega(1-k)}
\end{array}\right] \cdot\left[\begin{array}{c}
1 \\
J_{1} \\
Q_{1}
\end{array}\right]=\frac{\left[\begin{array}{c}
\rho \\
T \\
U
\end{array}\right]}{\left[\begin{array}{c}
P \cdot H \\
P \cdot H \\
P \cdot U
\end{array}\right],}
$$


where $\left\{\begin{array}{l}\rho_{Z}=\mathrm{i} \cdot \frac{(1-\gamma)(1+k)}{v(1-k)} \cdot \exp \left[\frac{z}{2 H}(1-k)\right], \\ T_{Z}=\mathrm{i} \cdot \frac{(1-\gamma)}{v} \cdot \exp \left[\frac{Z}{2 H}(1-k)\right], \\ U_{Z}=\mathrm{i} \cdot \frac{R T_{0}(1-\gamma) \sqrt{\varepsilon_{n}^{m}}}{a \Omega v(1-k)} \cdot \exp \left[\frac{Z}{2 H}(1-k)\right] .\end{array}\right.$

Equation (A26) is in accordance with Equation (2) in this work. In Equation (A26), $J_{1}$ is the source of the atmospheric tides, which are analyzed herein. In general, we can use Equation (A27) to calculate $J_{1}$.

\section{Acknowledgments}

The NCEP CFSR data were provided through the website http://rda.ucar.edu/datasets/ds093.1. The MEl data were supplied through the website http://www.esrl.noaa.gov/psd/enso/mei/. The present work is supported by the Strategic Priority Research Program of the Chinese Academy of Sciences (grant no. XDA17010201) and the National Science Foundation of China (grant nos. 41604138, 41427901, 41621063, 41474133, 41674158, 41874179, and 41322030).

\section{References}

Chapman, S., and Lindzen, R. S. (1970). Atmospheric Tides: Thermal and Gravitational. Dordrecht, Netherlands: D. Reidel.

Chen, Z. Y., and Lu, D. R. (2009a). Global structures of the DE3 tide. Chin. Sci. Bull., 54(6), 1073-1079. https://doi.org/10.1007/s11434-008-0585-x

Chen, Z. Y., and Lu, D. R. (2009b). On the calculation of Hough functions for resolving atmospheric thermal tidal structure. Chinese J. Geophys. (in Chinese), 52(6), 1444-1450. https://doi.org/10.3969/j.issn.00015733.2009.06.004

Forbes, J. M., and Garrett, H. B. (1978). Thermal excitation of atmospheric tides due to insolation absorption by $\mathrm{O}_{3}$ and $\mathrm{H}_{2} \mathrm{O}$. Geophys. Res. Lett., 5(12), 1013-1016. https://doi.org/10.1029/GL005i012p01013

Forbes, J. M., and Garrett, H. B. (1979). Theoretical studies of atmospheric tides. Rev. Geophys., 17(8), 1951-1981. https://doi.org/10.1029/RG017i008p01951

Forbes, J. M. (1995). Tidal and planetary waves. In R. M. Johnson, et al. (Eds.), The Upper Mesosphere and Lower Thermosphere: A Review of Experiment and Theory (pp. 67-87). Washington: American Geophysical Union. https://doi.org/10.1029/GM087p0067

Forbes, J. M., Zhang, X. L., Talaat, E. R., and Ward, W. (2003). Nonmigrating diurnal tides in the thermosphere. J. Geophys. Res., 108(A1), 1033. https://doi.org/10.1029/2002JA009262

Forbes, J. M., and Wu, D. (2006). Solar tides as revealed by measurements of mesosphere temperature by the MLS experiment on UARS. J. Atmos. Sci. 63(7), 1776-1797. https://doi.org/10.1175/jas3724.1

Gurubaran, S., Rajaram, R., Nakamura, T., and Tsuda, T. (2005). Interannual variability of diurnal tide in the tropical mesopause region: A signature of the El Nino-Southern Oscillation (ENSO). Geophys. Res. Lett., 32(13), L13805. https://doi.org/10.1029/2005GL022928

Hagan, M. E. (1996). Comparative effects of migrating solar sources on tidal signatures in the middle and upper atmosphere. J. Geophys. Res. Atmos., 101(D16), 21213-21222. https://doi.org/10.1029/96jd01374

Hagan, M. E., and Roble, R. G. (2001). Modeling diurnal tidal variability with the National Center for Atmospheric Research thermosphere-ionosphere-mesosphere-electrodynamics general circulation model. J. Geophys. Res. Space Phys., 106(A11), 24869-24882. https://doi.org/10.1029/2001ja000057

Hagan, M. E., and Forbes, J. M. (2002). Migrating and nonmigrating diurnal tides in the middle and upper atmosphere excited by tropospheric latent heat release. J. Geophys. Res. Atmos., 107(D24), ACL 6-1-ACL 6-15. https://doi.org/10.1029/2001JD001236
Hamilton, K. (1981). Latent heat release as a possible forcing mechanism for atmospheric tides. Mon. Wea. Rev., 109(1), 3-17. https://doi.org/10.1175/1520-0493(1981)109<0003:Ihraap>2.0.co;2

Harris, I., and Mayr, H. G. (1975). Diurnal variations in the thermosphere 1. Theoretical formulation. J. Geophys. Res., 80(28), 3925-3933. https://doi.org/10.1029/JA080i028p03925

Kato, S. (1980). Dynamics of the Upper Atmosphere. Tokyo: Center for Academic Publications Japan.

Li, X., Wan, W. X., Ren, Z. P., Liu, L. B., and Ning, B. Q. (2015a). The variability of nonmigrating tides detected from TIMED/SABER observations. J. Geophys. Res. Space Phys., 120(12), 10793-10808. https://doi.org/10.1002/2015JA021577

Li, X., Wan, W. X., Yu, Y., and Ren, Z. P. (2015b). Yearly variations of the stratospheric tides seen in the CFSR reanalysis data. Adv. Space Res., 56(9), 1822-1832. https://doi.org/10.1016/j.asr.2015.01.014

Lieberman, R. S., Riggin, D. M., Ortland, D. A., Nesbitt, S. W., and Vincent, R. A. (2007). Variability of mesospheric diurnal tides and tropospheric diurnal heating during 1997-1998. J. Geophys. Res. Atmos., 112(D20), D20110. https://doi.org/10.1029/2007JD008578

Lindzen, R. S., and Blake, D. (1970). Mean heating of the thermosphere by tides. J. Geophys. Res., 75(33), 6868-6871. https://doi.org/10.1029/JC075i033p06868

Lindzen, R. S., and Will, D. I. (1973). An analytic formula for heating due to ozone absorption. J. Atmos. Sci., 30(3), 513-515. https://doi.org/10.1175/15200469(1973)030<0513:AAFFHD>2.0.CO;2

Manson, A. H., Luo, Y., and Meek, C. (2002). Global distributions of diurnal and semi-diurnal tides: observations from HRDI-UARS of the MLT region. Ann. Geophys., 20(11), 1877-1890. https://doi.org/10.5194/angeo-20-1877-2002

Manzini, E. (2009). Atmospheric science: ENSO and the stratosphere. Nat. Geosci., 2(11), 749-750. https://doi.org/10.1038/ngeo677

Mayr, H. G., and Harris, I. (1977). Diurnal variations in the thermosphere, 2. Temperature, composition, and winds. J. Geophys. Res., 82(19), 2628-2640. https://doi.org/10.1029/JA082i019p02628

McLandress, C., and Ward, W. E. (1994). Tidal/gravity wave interactions and their influence on the large-scale dynamics of the middle atmosphere: Model results. J. Geophys. Res. Atmos., 99(D4), 8139-8155. https://doi.org/10.1029/94JD00486

Oberheide, J., Hagan, M. E., Roble, R. G., and Offermann, D. (2002). Sources of nonmigrating tides in the tropical middle atmosphere. J. Geophys. Res. Atmos., 107(D21), ACL 6-1-ACL 6-14. https://doi.org/10.1029/2002jd002220

Oberheide, J., Forbes, J. M., Häusler, K., Wu, Q., and Bruinsma, S. L. (2009). Tropospheric tides from 80 to $400 \mathrm{~km}$ : Propagation, interannual variability, and solar cycle effects. J. Geophys. Res. Atmos., 114(D1), D00I05. https://doi.org/10.1029/2009JD012388

Oberheide, J., Forbes, J. M., Zhang, X., and Bruinsma, S. L. (2011). Wave-driven variability in the ionosphere-thermosphere-mesosphere system from TIMED observations: What contributes to the "wave 4"? J. Geophys. Res. Space Phys., 116(A1), A01306. https://doi.org/10.1029/2010ja015911

Pancheva, D., Mukhtarov, P., and Andonov, B. (2010). Global structure, seasonal and interannual variability of the eastward propagating tides seen in the SABER/TIMED temperatures (2002-2007). Adv. Space Res., 46(3), 257-274. https://doi.org/10.1016/j.asr.2010.03.026

Randel, W. J., Garcia, R. R., Calvo, N., and Marsh, D. (2009). ENSO influence on zonal mean temperature and ozone in the tropical lower stratosphere. Geophys. Res. Lett., 36(15), L15822. https://doi.org/10.1029/2009gl039343

Ren, Z. P., Wan, W. X., Liu, L. B., Zhao, B. Q., Wei, Y., Yue, X. N., and Heelis, R. A. (2008). Longitudinal variations of electron temperature and total ion density in the sunset equatorial topside ionosphere. Geophys. Res. Lett., 35(5), L05108. https://doi.org/10.1029/2007GL032998

Saha, S., Moorthi, S., Pan, H. L., Wu, X. R., Wang, J. D., Nadiga, S., Tripp, P., Kistler, R., Woollen, J., ... Goldberg, M. (2010). The NCEP climate forecast system reanalysis. Bull. Amer. Meteor. Soc., 91(8), 1015-1058. https://doi.org/10.1175/2010Bams3001.1

Sakazaki, T., Fujiwara, M., Zhang, X., Hagan, M. E., and Forbes, J. M. (2012). Diurnal tides from the troposphere to the lower mesosphere as deduced from TIMED/SABER satellite data and six global reanalysis data sets. $J$. 
Geophys. Res. Atmos., $117(\mathrm{D} 13), \mathrm{D} 13108$. https://doi.org/10.1029/2011JD017117

Svoboda, A. A., Forbes, J. M., and Miyahara, S. (2005). A space-based climatology of diurnal MLT tidal winds, temperatures and densities from UARS wind measurements. J. Atmos. Sol. Terr. Phys., 67(16), 1533-1543. https://doi.org/10.1016/j.jastp.2005.08.018

Talaat, E. R. and Lieberman, R. S. (1999). Nonmigrating diurnal tides in mesospheric and lower-thermospheric winds and temperatures. J. Atmos. Sci., 56(24), 4073-4087. https://doi.org/10.1175/1520-0469

Trenberth, K. E., Caron, J. M., Stepaniak, D. P., and Worley, S. (2002). Evolution of El Niño - Southern Oscillation and global atmospheric surface temperatures. J. Geophys. Res., 107(D8), 4065. https://doi.org/10.1029/2000JD000298

Vial, F., Lott, F., and Teitelbaum, H. (1994). A possible signal of the El Niño Southern Oscillation in time series of the diurnal tide. Geophys. Res. Lett., 21(15), 1603-1606. https://doi.org/10.1029/94GL01016

Volland, H. (1988). Atmospheric Tidal and Planetary Waves. Dordrecht: Kluwer Academic Publishers.

Wan, W., Liu, L., Pi, X., Zhang, M. L., Ning, B., Xiong, J., and Ding, F. (2008).
Wavenumber-4 patterns of the total electron content over the low latitude ionosphere. Geophys. Res. Lett., 35(12), L12104.

https://doi.org/10.1029/2008GL033755

Wan, W., Xiong, J., Ren, Z., Liu, L., Zhang, M. L., Ding, F., Ning, B., Zhao, B., and Yue, X. (2010). Correlation between the ionospheric WN4 signature and the upper atmospheric DE3 tide. J. Geophys. Res. Space Phys., 115(A11), A11303. https://doi.org/10.1029/2010JA015527

Yu, Y., Wan, W. X., Ning, B. Q., Liu, L. B., Wang, Z. G., Hu, L. H., and Ren, Z. P. (2013). Tidal wind mapping from observations of a meteor radar chain in December 2011. J. Geophys. Res. Space Phys., 118(5), 2321-2332. https://doi.org/10.1029/2012JA017976

Yu, Y., Wan, W. X., Ren, Z. P., Xiong, B., Zhang, Y., Hu, L. H., Ning, B., and Liu, L. B. (2015). Seasonal variations of MLT tides revealed by a meteor radar chain based on Hough mode decomposition. J. Geophys. Res. Space Phys., 120(8), 7030-7048. https://doi.org/10.1002/2015JA021276

Zhang, X. L., Forbes, J. M., Hagan, M. E., Russell III, J. M., Palo, S. E., Mertens, C. J., and Mlynczak, M. G. (2006). Monthly tidal temperatures 20-120 km from TIMED/SABER. J. Geophys. Res. Space Phys., 111(A10), A10S08. https://doi.org/10.1029/2005JA011504 